\title{
The quality of reporting methods and results of cost-effectiveness analyses in Spain: a methodological systematic review
}

Ferrán Catalá-López ${ }^{1,2,3^{*} \dagger}$, Manuel Ridao ${ }^{4 \dagger}$, Adolfo Alonso-Arroyo ${ }^{5}$, Anna García-Altés ${ }^{1,6}$, Chris Cameron ${ }^{3,7}$, Diana González-Bermejo ${ }^{8}$, Rafael Aleixandre-Benavent ${ }^{9}$, Enrique Bernal-Delgado ${ }^{4}$, Salvador Peiró ${ }^{10}$, Rafael Tabarés-Seisdedos ${ }^{2}$ and Brian Hutton ${ }^{3,11}$

\begin{abstract}
Background: Cost-effectiveness analysis has been recognized as an important tool to determine the efficiency of healthcare interventions and services. There is a need for evaluating the reporting of methods and results of costeffectiveness analyses and establishing their validity. We describe and examine reporting characteristics of methods and results of cost-effectiveness analyses conducted in Spain during more than two decades.

Methods: A methodological systematic review was conducted with the information obtained through an updated literature review in PubMed and complementary databases (e.g. Scopus, ISI Web of Science, National Health Service Economic Evaluation Database (NHS EED) and Health Technology Assessment (HTA) databases from Centre for Reviews and Dissemination (CRD), Índice Médico Español (IME) Índice Bibliográfico Español en Ciencias de la Salud (IBECS)). We identified cost-effectiveness analyses conducted in Spain that used quality-adjusted life years (QALYs) as outcome measures (period 1989-December 2014). Two reviewers independently extracted the data from each paper. The data were analysed descriptively.

Results: In total, 223 studies were included. Very few studies (10; $4.5 \%)$ reported working from a protocol. Most studies (200; $89.7 \%$ ) were simulation models and included a median of 1000 patients. Only 105 (47.1\%) studies presented an adequate description of the characteristics of the target population. Most study interventions were categorized as therapeutic $(189 ; 84.8 \%)$ and nearly half $(111 ; 49.8 \%)$ considered an active alternative as the comparator. Effectiveness of data was derived from a single study in 87 (39.0\%) reports, and only few (40; 17.9\%) used evidence synthesis-based estimates. Few studies (42; $18.8 \%$ ) reported a full description of methods for QALY calculation. The majority of the studies $(147 ; 65.9 \%)$ reported that the study intervention produced "more costs and more QALYs" than the comparator. Most studies (200; $89.7 \%$ ) reported favourable conclusions. Main funding source was the private for-profit sector (135; $60.5 \%$ ). Conflicts of interest were not disclosed in 88 (39.5\%) studies.

Conclusions: This methodological review reflects that reporting of several important aspects of methods and results are frequently missing in published cost-effectiveness analyses. Without full and transparent reporting of how studies were designed and conducted, it is difficult to assess the validity of study findings and conclusions.
\end{abstract}

Keywords: Cost-effectiveness analysis, Cost-utility analysis, Quality-adjusted life years, Methodology, Reporting, Spain

\footnotetext{
* Correspondence: ferran_catala@hotmail.com

${ }^{\dagger}$ Equal contributors

${ }^{1}$ Fundación Instituto de Investigación en Servicios de Salud, Valencia, Spain

${ }^{2}$ Department of Medicine, University of Valencia/INCLIVA Health Research

Institute and CIBERSAM, Valencia, Spain

Full list of author information is available at the end of the article
} 


\section{Background}

Cost-effectiveness analysis has been recognized as an important tool to assist clinicians, scientists and policymakers in determining the efficiency of healthcare interventions, guiding societal decision-making on the financing of healthcare services and establishing research priorities. Given that the information provided by cost-effectiveness analysis has the potential to impact population health and health services, there is a need for evaluating the reporting of methods and results of cost-effectiveness analyses and establishing their validity to inform policymaking [1-4].

Diverse approaches to synthesize evidence have been considered in biomedical research [5-8], including economic evaluations of healthcare interventions [9-16]. At the same time, decision-making in health care requires an understanding of the state of economic evaluation at a national level, where the completeness of the reporting is generally less well understood but where specific priorities are often set. As a way of understanding the maturity and growth of the field, several smaller studies have examined a limited set of reporting characteristics of cost-effectiveness analyses published in Spain [17-20]. Spain was a pioneer in proposing the standardization and reporting of methodology applicable to costeffectiveness analysis [21, 22]. However, the institutional and regulatory framework has so far not helped the application of the methodology to the public health decisions. The central government of Spain is the main decision-maker in pricing and reimbursement related to new medicines and healthcare technologies, although with a high decentralization of health jurisdictions in several regional health services, but traditionally, there have been no national requirements related to the costeffectiveness for making coverage decisions.

We present herein a case study about reporting practices of economic evaluations of healthcare interventions in one Western European country: Spain. Specifically, this study expands upon previous research [23, 24] to comprehensively describe and examine reporting characteristics of methods and results of cost-effectiveness analyses conducted in Spain during more than two decades.

\section{Methods}

This methodological systematic review has been reported according to the Preferred Reporting Items for Systematic Reviews and Meta-Analyses (PRISMA) statement [25] (see Additional file 1: Table S3). A brief protocol was developed prior to the initiation of this review. It can be acquired by request from the corresponding authors. We did not register the protocol with PROSPERO given that the register does not accept methodological reviews.

\section{Literature search}

The results from a previous review that examined collaborative patterns of scientific production in a cohort of cost-effectiveness analyses conducted in Spain within the period 1989-2011 [23] were updated with the studies published until December 2014 and subsequently analysed. A systematic search was performed in PubMed/ MEDLINE and other databases such as Scopus, ISI Web of Science, National Health Service Economic Evaluation Database (NHS EED) and Health Technology Assessment (HTA) databases of the Centre for Reviews and Dissemination (CRD) at the University of York, UK, as well as Índice Médico Español (IME) and Índice Bibliográfico Español en Ciencias de la Salud (IBECS). The search included a broad range of terms related to economic evaluations of healthcare interventions, costeffectiveness analyses and the geographical area "Spain". For the section of geographical area, the search was based on a previously validated filter by Valderas [26] to minimize bias regarding the indexing of geographical items. This filter is constructed around three complementary approaches: (a) the term "Spain" and its variants in various languages; (b) related mainly to region and province place names and (c) acronyms for regional health services. PubMed/MEDLINE and the above mentioned complementary databases were searched from January 1, 2011 to December 31, 2014; the PubMed/ MEDLINE search strategy is provided in an online supplement to this review (see Additional file 1: Table S1). Furthermore, manual searches were made for publicly available reports from the Health Technology Agencies and publications in specialized Spanish journals.

\section{Inclusion criteria and study selection}

Our selection of studies was based on cost-effectiveness analyses of healthcare interventions that used qualityadjusted life years (QALYs) as outcome measure (see Table 1 for terminology). In the health economic

\section{Table 1 Terminology}

Cost-effectiveness analysis is a specific form of economic evaluation comparing two or more alternative programmes by measuring costs and consequences. Consequences are measured in natural units (e.g. life years gained or cases averted).

Cost-utility analysis is a variant of cost-effectiveness analysis, where consequences are measured in terms of summary measures of population health such as quality-adjusted life years.

Cost-effectiveness acceptability curve is a graphical representation of the cost-effectiveness comparison between two interventions and plots the probability that one intervention is more cost-effective than other, as a function of the willingness-to-pay threshold for one additional unit of benefits.

Incremental cost-effectiveness ratio (ICER) is the ratio of the change in costs of an intervention (compared to the alternative) to the change in effects of the intervention.

Quality-adjusted life years (QALYs) are a measure that combines length of life and quality of life in a single outcome. 
literature, this type of studies is sometimes known as "cost-utility analyses". We selected this type of costeffectiveness analyses because many scientists and policymakers have recommended the QALY framework as the standard reference for cost-effectiveness [27]. Studies had to be undertaken in the Spanish population. Review studies, editorials, and abstracts of congresses were excluded. If an article was found repeated in several publications, that published earlier (e.g. when there are two or more articles of the same study) and/or published in a journal with higher impact factor (e.g. when there exists a study published in both health technology assessment report and journal manuscript) was included.

All citations of potential relevance identified from the literature search were screened by one reviewer. Two reviewers reviewed all potentially relevant articles in full text. Final inclusion was confirmed if both reviewers felt the study was directly relevant to the objectives of this methodological review. Planned involvement of a third party to deal with unresolved discrepancies was not required.

\section{Data collection}

Two reviewers (with expertise in health economics and evidence synthesis) extracted data from each retrieved paper independently. Data were collected using a selfdeveloped item data collection form designed to assess reporting details of the studies. The process of data extraction was piloted in 20 records. A final data extraction form was then agreed. To enable description of the characteristics and the quality of reporting of costeffectiveness analyses in each report, we gathered the following information from all studies: year and journal of publication, impact factor (according to 2014 Journal Citation Report), country of first author, mention of a protocol, study objective, study design (e.g. randomized trial, observational study, simulation model), intervention targeted (e.g. prevention, diagnosis/prognosis, treatment, rehabilitation), type of comparators (e.g. active alternative, do nothing or placebo, usual care), perspective of analysis (in terms of which costs are considered, e.g. society, national healthcare system, hospital, others), type of costs (e.g. direct or indirect) and sources of information, the main cause of disease to which the intervention or health programme was addressed, description of population characteristics, time horizon, sources of clinical effectiveness (e.g. based on a single study or based on systematic reviews and meta-analyses), full description of methods for QALY calculation, discussion of assumptions and validation of models (if applicable), discount rates for costs and outcomes, results for the primary outcome in the base case scenario (e.g. "more costs, more QALYs", "less costs, more QALYs", "less costs, comparable QALYs"), incremental analyses including incremental cost-effectiveness ratios (ICERs), uncertainty measures (e.g. confidence intervals, acceptability curves), sensitivity analyses, limitations of study, comparison of results with those of other studies, hypothetical willingness-to-pay threshold and study conclusions. Conclusions reported in the published article were defined as follows: favourable if the intervention was clearly claimed to be the preferred choice (e.g. cited as "cost-effective", "reduced costs", "produced cost savings", "an affordable option", "value for money"); unfavourable if the final comments were negative (e.g. the intervention is "unlikely to be cost-effective", "produced higher costs", "is economically unattractive" or "exceeded conventional thresholds of willingness to pay"); and neutral or uncertain when the intervention of interest did not surpass the comparator and/or when some uncertainty was expressed in the conclusions. Disclosures of funding source, conflicts of interest and authors' contributions were also evaluated.

\section{Statistical analysis}

A descriptive analysis was performed using frequency and percentage counts. All calculations were performed using Stata (Version 13, StataCorp LP, College Station, TX, USA).

\section{Results \\ Search}

The flow diagram in Additional file 1: Figure S1 presents the process of study selection.

Eight out of 131 identified studies from the cohort of cost-effectiveness analysis conducted within the period 1989-2011 [23] were excluded for not meeting the defined criteria. Our updated search identified 2014 records. Initial screening excluded 1914 records. The remaining 100 full-text articles were assessed for additional scrutiny, of which 21 where ineligible. Complementary searches through other sources (e.g. publicly available reports from the Health Technology Agencies and publications in specialized Spanish journals) identified 21 additional studies and were added to the previously identified, obtaining a total sample of 223 studies (see Additional file 1: Table S2).

\section{General characteristics}

The 223 studies were published in 98 journals (206; $92.4 \%)$ or assessment reports by the health technology assessment agencies $(17 ; 7.6 \%)$. The majority of the journals published only one cost-effectiveness analysis although 15 journals each published four or more studies (Table 2). Most studies were published in journals with impact factors $\leq 5.0$ and only four studies were published in journals with impact factor $>10$. The number of studies increased exponentially over the study period 
Table 2 Characteristics of included cost-effectiveness analyses $(n=223)$

\begin{tabular}{|c|c|c|}
\hline Characteristic & Number & Percent \\
\hline \multicolumn{3}{|l|}{ Journals publishing } \\
\hline 1 paper & 60 & 61.2 \\
\hline 2 papers & 17 & 17.3 \\
\hline 3 papers & 6 & 6.1 \\
\hline 4 papers or more & 15 & 15.3 \\
\hline \multicolumn{3}{|l|}{ Papers by source } \\
\hline Journal articles & 206 & 92.4 \\
\hline Health technology assessment reports & 17 & 7.6 \\
\hline \multicolumn{3}{|l|}{ Papers by journal impact factor (JCR 2014) } \\
\hline None & 88 & 39.5 \\
\hline $0.1-2.0$ & 61 & 27.3 \\
\hline $2.1-5.0$ & 58 & 26.0 \\
\hline $5.1-10.0$ & 12 & 5.4 \\
\hline$>10.0$ & 4 & 1.8 \\
\hline \multicolumn{3}{|l|}{ Papers by language of publication } \\
\hline English & 127 & 57.0 \\
\hline Spanish & 96 & 43.0 \\
\hline \multicolumn{3}{|l|}{ Number of authors per paper } \\
\hline 1 & 3 & 1.3 \\
\hline $2-3$ & 33 & 14.8 \\
\hline $4-7$ & 143 & 64.8 \\
\hline$\geq 8$ & 44 & 19.7 \\
\hline \multicolumn{3}{|l|}{ Country of first author } \\
\hline Spain & 183 & 82.1 \\
\hline UK & 8 & 3.6 \\
\hline USA & 7 & 3.1 \\
\hline Italy & 5 & 2.2 \\
\hline The Netherlands & 4 & 1.8 \\
\hline Sweden & 4 & 1.8 \\
\hline Other & 12 & 5.4 \\
\hline \multicolumn{3}{|l|}{ Focus of interventions } \\
\hline Prevention & 18 & 8.1 \\
\hline Diagnosis/prognosis & 15 & 6.7 \\
\hline Treatment & 189 & 84.8 \\
\hline Rehabilitation & 1 & 0.4 \\
\hline \multicolumn{3}{|l|}{ Disease conditions } \\
\hline Cardiovascular diseases & 47 & 21.1 \\
\hline Malignant neoplasms & 36 & 16.1 \\
\hline Infectious diseases & 31 & 13.9 \\
\hline Neurological and mental disorders & 30 & 13.4 \\
\hline Musculoskeletal disorders & 20 & 9.0 \\
\hline Other conditions & 59 & 26.5 \\
\hline
\end{tabular}

JCR Journal Citation Report 2014
(Additional file 1: Figure S2), with nearly half of the cost-effectiveness analyses published during 2011-2014 (110; $49.3 \%)$. More than half $(127 ; 57.0 \%)$ of the reports were written in English. The studies included a median of six authors although 44 (19.7\%) were authored by eight or more authors and only $3(1.3 \%)$ reports were single authored. The majority of the interventions were classified as treatments $(189 ; 84.8 \%$ ) - of which more than $75 \%(143 / 189)$ were pharmaceuticals. Cardiovascular diseases (47; $21.1 \%)$ and malignant neoplasms (36; $16.1 \%)$ were the disease conditions most commonly studied.

\section{Reporting characteristics of methods and results}

Table 3 provides a summary of the descriptive and reporting characteristics of the included studies. The majority of the study reports used the specific terms "cost-effectiveness" or "cost-utility analysis" in the title $(181 ; 81.2 \%)$ and presented clearly the study question (187; $83.9 \%)$. However, only 10 studies (4.5\%) reported working from a protocol-of which 7 were randomized controlled trials, 2 were simulation models and 1 was an observational study.

Of the identified studies, 200 (89.7\%) were model-based being Markov models as the most frequently reported (135; $60.5 \%)$. A minimal number of non-model-based studies were randomized controlled trials $(10 ; 4.5 \%)$.

Overall, most of the analyses were conducted in the adult population $(170 ; 76.2 \%)$ but only $105(47.1 \%)$ presented an adequate description of the characteristics of the base case population or identified the indication clearly. The studies reporting the sample size (127; 57.0 \%) included a median of 1000 patients (25th percentile $=301$; 75th percentile $=10000$ ), although this number varied considerably by the type of the study (e.g. clinical trials, median $=115$ patients; observational studies, median = 200 patients; and simulation models, median $=1000$ patients). Most of the studies included an adequate description of the interventions and comparators (184; $82.5 \%$ ). Nearly half $(111 ; 49.8 \%)$ of the studies considered an active alternative as the comparator (e.g. drug, device, procedure, programme), 73 (32.7\%) used usual care and 39 (17.5\%) placebos or "do nothing". The study perspective was clearly stated in most of the analyses (207; $92.8 \%$ ). The national healthcare system perspective was the most commonly used (156; $70.0 \%$ ).

The time horizon was clearly reported in the majority of studies (218; $97.8 \%)$. Overall, 174 studies (78.0\%) used a time horizon greater than 1 year.

Most studies (178; $79.8 \%$ ) reported on the diagram of modelling or flow of patients (e.g. in the case of randomized controlled trials and observational studies). Most studies (172; $77.1 \%$ ) reported on the assumptions adopted for the analyses. Regarding the simulation and 
Table 3 Descriptive and reporting characteristics of included cost-effectiveness analyses $(n=223)$

\begin{tabular}{|c|c|c|c|}
\hline Category & Characteristic & Number & Percen \\
\hline \multirow[t]{2}{*}{ Title } & Identification & & \\
\hline & Specific terms "cost-effectiveness" or "cost-utility analysis" in title & 181 & 81.2 \\
\hline \multirow[t]{2}{*}{ Objective } & Study question & & \\
\hline & Clear presentation of study question and its relevance for decision-making & 187 & 83.9 \\
\hline \multirow[t]{19}{*}{ Methods } & Protocol & & \\
\hline & Existence of study protocol (or a priori established methods) & 10 & 4.5 \\
\hline & Type of study & & \\
\hline & Model based & 200 & 89.7 \\
\hline & Deterministic decision-tree model & 29 & 13.0 \\
\hline & Markov model & 135 & 60.5 \\
\hline & Discrete event simulation & 11 & 4.9 \\
\hline & Other (or unclear) & 25 & 11.2 \\
\hline & Non-model based & 23 & 10.3 \\
\hline & Observational (non-interventional) study & 13 & 5.8 \\
\hline & Randomized controlled trial & 10 & 4.5 \\
\hline & Population & & \\
\hline & Number of participants included (or simulated) & 127 & 57.0 \\
\hline & Adequate description of characteristics of the base case population & 105 & 47.1 \\
\hline & Adults & 170 & 76.2 \\
\hline & Children and adolescents & 11 & 4.9 \\
\hline & Newborn and infants (less than 1 year) & 8 & 3.5 \\
\hline & Overall population & 4 & 1.8 \\
\hline & Not reported & 30 & 13.5 \\
\hline
\end{tabular}

Type of interventions

$\begin{array}{lr}\text { Pharmaceuticals } & 143\end{array}$

Device/procedure

Screening

Surgery

Educational/behavioural

Other

Type of comparators

Active alternative

Usual care

Placebo or do nothing

Adequate description of interventions and comparators

Study perspective clearly stated

National Health System only

National Health System and societal

Societal only

Hospital

Time horizon reported 
Table 3 Descriptive and reporting characteristics of included cost-effectiveness analyses $(n=223)$ (Continued)

\begin{tabular}{|c|c|c|c|}
\hline & Assumptions discussed & 172 & 77.1 \\
\hline & Model validation discussed (when applicable) & 88 & 44.0 \\
\hline & Reasons for the specific model used (when applicable) & 91 & 45.5 \\
\hline & \multicolumn{3}{|l|}{ Measurement of effectiveness ${ }^{a}$} \\
\hline & Based on a single study & 87 & 39.0 \\
\hline & Based on evidence synthesis (e.g. systematic review and/or meta-analysis) & 40 & 17.9 \\
\hline & Full description of QALY calculation & 42 & 18.8 \\
\hline & Harms were considered & 129 & 57.8 \\
\hline & \multicolumn{3}{|l|}{ Cost and resources information } \\
\hline & Source of valuation for all cost items reported & 216 & 96.9 \\
\hline & Quantity of resources & 107 & 48.0 \\
\hline & Year of monetary units & 195 & 87.4 \\
\hline & \multicolumn{3}{|l|}{ Costing } \\
\hline & Direct costs only & 182 & 81.6 \\
\hline & Direct and indirect costs & 41 & 18.4 \\
\hline & Discount rate for costs and QALYs & 161 & 72.2 \\
\hline \multirow[t]{22}{*}{ Results } & Net costs reported & 197 & 88.3 \\
\hline & Net benefits reported & 192 & 86.1 \\
\hline & Incremental cost-effectiveness ratio (ICER) reported & 207 & 92.8 \\
\hline & Confidence intervals (e.g. 95 \% Cl) & 27 & 12.1 \\
\hline & Cost-effectiveness plane & 99 & 44.4 \\
\hline & Acceptability curves & 92 & 41.3 \\
\hline & Sensitivity analysis reported & 201 & 90.1 \\
\hline & For costs & 170 & 76.2 \\
\hline & For estimates of effectiveness & 158 & 70.9 \\
\hline & For utility weights & 95 & 42.6 \\
\hline & For discount rates & 82 & 36.8 \\
\hline & Type of sensitivity analysis & & \\
\hline & Deterministic univariate & 85 & 38.1 \\
\hline & Deterministic multivariate & 6 & 2.7 \\
\hline & Probabilistic & 110 & 49.3 \\
\hline & Results for the primary outcome in the base case scenario & & \\
\hline & More costs, more QALYs & 147 & 65.9 \\
\hline & Less costs, more QALYs & 63 & 28.3 \\
\hline & Less costs, comparable QALYs & 5 & 2.2 \\
\hline & More costs, comparable QALYs & 4 & 1.8 \\
\hline & Less costs, less QALYs & 2 & 0.9 \\
\hline & Comparable costs, more QALYs & 2 & 0.9 \\
\hline \multirow[t]{7}{*}{ Discussion } & Limitations of study discussed & 197 & 88.3 \\
\hline & Results compared with those of other economic evaluations & 165 & 74.0 \\
\hline & \multicolumn{3}{|l|}{ Hypothetical willingness-to-pay (WTP) threshold reported } \\
\hline & $<30,000 € / Q A L Y$ & 4 & 1.8 \\
\hline & $30,000 € / Q A L Y$ & 126 & 56.5 \\
\hline & $>30,000 € / Q A L Y-\leq 50,000 € / Q A L Y$ & 36 & 16.1 \\
\hline & $>50,000 € / Q A L Y$ & 7 & 3.1 \\
\hline
\end{tabular}


Table 3 Descriptive and reporting characteristics of included cost-effectiveness analyses $(n=223)$ (Continued)

\begin{tabular}{|c|c|c|c|}
\hline \multirow{2}{*}{\multicolumn{2}{|c|}{$\begin{array}{l}\text { Unclear or not reported } \\
\text { Study conclusions }\end{array}$}} & 50 & 22.4 \\
\hline & & & \\
\hline & Favourable & 200 & 89.7 \\
\hline & Unfavourable & 12 & 5.4 \\
\hline & Neutral/unclear & 11 & 4.9 \\
\hline \multirow[t]{9}{*}{ Other } & Disclosed funding sources & 169 & 75.8 \\
\hline & Private/for profit & 135 & 60.5 \\
\hline & Public & 38 & 17.0 \\
\hline & None/not reported & 49 & 22.0 \\
\hline & Mixed & 1 & 0.4 \\
\hline & Disclosed conflicts of interest & 135 & 60.5 \\
\hline & With conflicts of interest & 94 & 42.1 \\
\hline & With no conflicts of interest & 41 & 18.4 \\
\hline & Disclosed authors' contribution & 46 & 20.6 \\
\hline
\end{tabular}

${ }^{a}$ Measurement of effectiveness only relates to effect estimates. The epidemiology of disease and/or transition probabilities were not necessarily based on a systematic review and meta-analysis

modelling-based studies, nearly half reported reasons for the specific model used (91/200; $45.5 \%$ ) and/or provided some information on the model validation (88/200; $44.0 \%)$ such as previous publication in other settings.

Effectiveness of data was derived from a single study in 87 (39.0 \%) analyses. Only 40 (17.9 \%) used evidence synthesis-based estimates (e.g. systematic reviews and meta-analyses).

The methods that were reported for calculating QALYs are detailed in Table 4. Overall, a small number of the studies (42; $18.8 \%$ ) reported a full description of methods for QALY calculation. About half of the studies (109; $48.9 \%)$ reported information on the health-state classification system, of which the EuroQoL-5D was the instrument most commonly reported (82; $36.8 \%$ ). Half of the studies $(115 ; 51.6 \%)$ provided the source of the preferences. Most frequently, the patients and their caregivers $(103 ; 46.2 \%)$ were the source. Only a small number of the studies $(43 ; 19.3 \%)$ provided information on the measurement technique used for valuing health

Table 4 Descriptive and reporting characteristics of methods used in calculating QALYs

\begin{tabular}{|c|c|c|c|}
\hline Category & Characteristic & Number & Percent \\
\hline \multirow[t]{6}{*}{ Health-state classification system } & EuroQoL-5D & 82 & 36.8 \\
\hline & SF-36 & 6 & 2.7 \\
\hline & Rosser scale & 6 & 2.7 \\
\hline & Health Utility Index (HUI) & 2 & 0.9 \\
\hline & Other & 13 & 5.8 \\
\hline & Not reported & 114 & 51.1 \\
\hline \multirow[t]{4}{*}{ Source of preferences } & Patient/caregiver & 103 & 46.2 \\
\hline & Community & 10 & 4.5 \\
\hline & Clinician/author & 2 & 0.9 \\
\hline & Not reported & 108 & 48.4 \\
\hline \multirow[t]{5}{*}{ Measurement technique used for valuing health state } & Time tradeoff (TTO) & 22 & 9.9 \\
\hline & Visual analogue scale (VAS) & 12 & 5.4 \\
\hline & Standard gamble (SG) & 5 & 2.2 \\
\hline & Tariffs for classification & 4 & 1.8 \\
\hline & Not reported & 182 & 81.6 \\
\hline \multirow[t]{3}{*}{ Country/region of reference for utility weights } & National/local population (e.g. Spain) & 50 & 22.4 \\
\hline & Citation of the international literature & 143 & 64.1 \\
\hline & Not reported & 30 & 13.5 \\
\hline
\end{tabular}


states. The time tradeoff $(22 ; 9.9 \%)$ was the most commonly used technique. The majority of the studies used the published international literature for data on utility weights $(143$; $64.1 \%)$ and only 50 studies $(22.4 \%)$ reported country-specific utility weights for Spain.

Half of the studies $(129 ; 57.8 \%)$ reported on some aspect of harms.

Ninety-seven percent (216) of the studies identified sources of valuation for costing items, and $87.4 \%$ (195) indicated the year of currency. Overall, 107 (48.0 \%) studies described quantity of resources. Eighteen percent (41) of studies included indirect costs. Seventy-two percent (161) of studies discounted both costs and QALYs. Of the studies with a time horizon greater than 1 year (Table 5), the most commonly used was a $3 \%$ discount rate.

In terms of results (Table 3), most of the studies (207; $92.8 \%$ ) reported ICERs (median $=16,908 €$; 25 th percentile $=8,998 € ; 75$ th percentile $=38,000 €)$. However, few studies $(27 ; 12.1 \%)$ described point estimates together with an associated confidence interval. Nearly half of the studies $(99 ; 44.4 \%)$ reported the costeffectiveness plane. Similarly, less than half of the studies (92; $41.3 \%$ ) reported a willingness-to-pay curve ("costeffectiveness acceptability curve") to contrast the results of the analyses against an arbitrary efficiency threshold. Overall, $90.1 \%$ (201) of studies reported sensitivity analyses. About half of the studies (110; $49.3 \%$ ) conducted a probabilistic sensitivity analysis. The majority of the studies $(147 ; 65.9 \%)$ reported that the study intervention produced "more costs and more QALYs" than the alternative comparator for the primary outcome of the base case scenario. Sixty-three $(28.3 \%)$ studies reported that the intervention was a dominant strategy, that means that the study intervention was "more effective and less costly" than the alternative.

Overall, the vast majority of the studies (197; $88.3 \%)$ discussed limitations of the analyses. Most studies (165; $74.0 \%)$ compared their results with those of previous

Table 5 Discount rates used in included cost-effectiveness analyses

\begin{tabular}{lllll}
\hline & Costs & & QALYs \\
Discount rate (\%) & Number & Percent & Number & Percent \\
\hline 1.5 & - & - & 3 & 1.7 \\
2 & 1 & 0.6 & 1 & 0.6 \\
3 & 107 & 61.5 & 109 & 62.6 \\
3.5 & 31 & 17.8 & 31 & 17.8 \\
4 & 1 & 0.6 & 1 & 0.6 \\
5 & 5 & 2.9 & 4 & 2.3 \\
6 & 13 & 7.5 & 11 & 4.9 \\
Total $^{a}$ & 174 & 100.0 & 174 & 100.0 \\
\hline
\end{tabular}

ancludes cost-effectiveness analyses that investigated costs or QALYs over time horizons of more than 1 year economic analyses. About half of the studies (126; $56.5 \%)$ mentioned a hypothetical willingness-to-pay threshold of $30,000 € /$ QALY. The majority of studies (200; $89.7 \%)$ reported favourable conclusions for the primary outcomes. Only a minority $(12 ; 5.4 \%)$ of published cost-effectiveness analyses reported unfavourable conclusions. About three fourths (169; $75.8 \%)$ reported funding sources, being the private for-profit sector the main source $(135 ; 60.5 \%)$. Conflicts of interest were not disclosed in 88 (39.5\%) studies. Authors' contributions were only reported in 46 (20.6\%) studies (Table 3$)$.

\section{Discussion}

In this methodological systematic review, we identified 223 reports of cost-effectiveness analyses conducted in Spain over the period 1989-2014. Overall, the studies covered a wide range of disease conditions but predominantly addressed questions about the efficiency of therapeutic interventions. Our review, as well as other previously published reviews [11-14], showed that the quality of reporting of cost-effectiveness analyses varies widely and, in many cases, essential components of reporting methods and results were missing in published reports, such as the use of study protocols, the adequate description of patient characteristics, the measurement of clinical effectiveness using a systematic review process or the adequate description of QALY calculation.

Our study suggests the need for improvement in several aspects of published cost-effectiveness analyses. An important element in assessing research conduct and reporting is the study protocol. As showed in this review, only $4 \%$ of studies reported working from a protocol. Study protocols play an essential role in planning, conduct, interpretation, and external review of primary studies, but also in evidence synthesis of primary research. For example, the preparation and publication of a well-written protocol may reduce arbitrariness in decision-making when extracting and using data from primary research for populating health economic models. When clearly reported protocols are made available, they enable readers to identify deviations from planned methods and whether they bias the interpretation of results and conclusions [28, 29]. International registries (such as ClinicalTrials.gov for clinical trials and PROSPERO for systematic reviews and metaanalysis) are now a reality. Similarly, in recent years, reporting guidelines for protocols have been endorsed and implemented (e.g. Standard Protocol Items: Recommendations for Interventional Trials (SPIRIT) for protocols of clinical trials [28] and PRISMA for protocols (PRISMA-P) of systematic reviews [29]). However, in view of our results, this revolution has not occurred yet in the field of cost-effectiveness research and, thus, could warrant further pragmatic action. 
Many cost-effectiveness analyses (about $53 \%$ ) did not report detailed information on baseline clinical characteristics (e.g. eligibility and exclusion criteria of participants, the severity of disease, the stage in the natural history of the disease, comorbidities). Inadequate reporting of the characteristics of the target population is a far greater barrier to the assessment of the study's generalizability (applicability) and relevance to decision-making [30-32]. It is possible that this poor reporting reflects a major problem in secondary publications, such as many cost-effectiveness analyses using simulation models. Given that a clear understanding of these elements is required to judge to whom the results of a study apply (as the Consolidated Standards of Reporting Trials (CONSORT) [31, 32] statement underlines for randomized controlled trials), this information should also be provided in the report of cost-effectiveness analyses (e.g. in main text or in online supplement when allowed).

The vast majority (about $90 \%$ ) of published costeffectiveness analyses used decision modelling as the main methodology. Decision modelling is considered a methodological approach of evidence synthesis that reaches beyond the scope of systematic reviews and meta-analyses. It is essential for cost-effectiveness research to use all relevant evidence on the effectiveness of interventions under evaluation. Rarely will all relevant evidence come from a single study, and typically, it will have to be drawn from several clinical studies [33]. A disappointing result of this review is that few published studies reported the use of a systematic review process for the measurement of clinical effectiveness. While systematic reviews and meta-analyses are considered to be the gold standard in knowledge synthesis, only $18 \%$ of published cost-effectiveness analyses used evidence synthesis-based estimates of effect. Instead, $43 \%$ of the studies make arbitrary decisions about what studies to use to inform effectiveness data, whereas $39 \%$ of the studies reported that the effectiveness data derived from a single study (generally, without a clear description of why the single study was a sufficient source of all relevant clinical evidence). The use of QALYs is recognized as the main valuation technique to measure health outcomes in cost-effectiveness analyses. However, in our review, it was also troubling that few studies (19\%) reported a full description of methods for QALY estimation, thus potentially impairing confidence in the results and conclusions. Future studies should be transparent in reporting these important aspects.

Strong evidence of publication bias and other potential sources of bias have been reported in biomedical research [34-38]. For example, randomized controlled trials with "positive" findings are published more often, and more quickly, than trials with "negative" findings [37]. Similarly, empirical studies have detected that most published cost-effectiveness analyses report favourable findings [38]. In our review, very few published studies (about $10 \%$ ) reported unfavourable or neutral conclusions. Although it is somewhat premature to comment on this finding, this could be indicative of potential biases, such as publication bias or even potential screening a priori that may have been performed by the producers of studies, which would make that costeffectiveness analyses would have been only conducted in cases where a "positive" result was expected. In our opinion, this issue requires further investigation.

Several reporting guidelines are available and endorsed for many types of biomedical research [25, 28, 29, 31, 39] but also for cost-effectiveness analyses [40-46]. Such tools promote the consistent reporting of a minimal set of information for scientists and researchers reporting studies and the editors and peer reviewers assessing them for publication. Endorsement of reporting guidelines by journals for randomized controlled trials [47] and systematic reviews [48] has been shown to improve the quality of reporting. The incorporation of reporting guidelines within the peer-review process could potentially contribute to improvements in the quality of reports of cost-effectiveness analyses. On this regard, the Consolidated Health Economic Evaluation Reporting Standards (CHEERS) statement [40] has been proposed as an attempt to consolidate and update previous efforts [41-46] into a single useful reporting guideline for costeffectiveness research. Authors, peer reviewers and editors can promote reporting guideline endorsement and implementation as an important way to improve transparency and completeness of what they published, reducing waste in reporting research and increasing value $[49,50]$ of cost-effectiveness research.

Our study has several limitations. First, although the review has been drawn from an exhaustive review of original reports of cost-effectiveness analyses, it is possible that the search missed some articles with relevant elements or that some studies conducted may not have been published. In addition, for some reports repeated in several publications, our approach was the inclusion of those published in a journal with higher impact factor and/or published earlier [23, 24]. Thus, the decision to use report level instead treating the study as the unit of analysis may have limited the collection of all the reporting characteristics from multiple reports of the same study (where there exist). Second, we restricted our analysis to cost-effectiveness analyses that used QALYs as health outcome measure (namely, cost-utility analyses). In a previous descriptive analysis of economic evaluations conducted in Spain [24], only about $15 \%$ are costutility analyses. It would be interesting to explore whether other forms of economic evaluations using alternative outcome measures results in similar reporting 
patterns. Third, we relied upon the expertise and experience of our authorship team and on existing documents $[16,22]$ to identify core items related to the conduct and reporting that we would like to see (in the position of potential readers) in any published cost-effectiveness analysis. Given the dynamic nature of research, some opportunities for future research and development could be the impact assessment of a specific reporting guideline (such as the CHEERS statement [40]) and/or local recommendations on the reporting quality of published studies [51]. Fourth, the extent of the reporting of costeffectiveness analyses was limited to the information publicly available in the corresponding report (and online data supplements when available). There were no further inquiries or attempts to verify the data sources and tools used in the studies and only information about reporting characteristics was taken into account in the review, without considering other possible sources (e.g. contacting authors and/or their sponsors).

\section{Conclusions}

We presented a national case study for more generalizable discussions about quality and transparency issues of reporting cost-effectiveness analyses, likely to be of interest to authors, peer reviewers and editors-but also research funders and regulators-both within and beyond Spain. Based on the existing evidence, several deficiencies in the reporting of important aspects of methods and results are apparent in published costeffectiveness analyses.

Our study raises challenges for increasing value and reducing waste in cost-effectiveness research. Without full and transparent reporting of how studies were designed and conducted, it is difficult to assess validity of study findings and conclusions of published studies. This review also reinforces the need to improve mechanisms of peer review and publication process of costeffectiveness research.

\section{Additional file}

Additional file 1: Reporting methods and results of CEA Spain_annex. Table S1. PubMed/MEDLINE search strategy. Table S2. Included studies. Table S3. PRISMA Checklist. Figure S1. Flow diagram for study selection. Figure S2. Growth in published cost-effectiveness analyses. Spain, 1989-2014.

\section{Abbreviations}

CHEERS: Consolidated Health Economic Evaluation Reporting Standards; CONSORT: Consolidated Standards of Reporting Trials; CRD: Centre for Reviews and Dissemination; HTA: Health Technology Assessment; HUI: Health Utility Index; IBECS: Índice Bibliográfico Español en Ciencias de la Salud; ICER: incremental cost-effectiveness ratio; IME: Índice Médico Español; JCR: Journal Citation Report; NHS EED: NHS Economic Evaluation Database; PRISMA: Preferred Reporting Items for Systematic Reviews and MetaAnalyses; PRISMA-P: Preferred Reporting Items for Systematic Reviews and Meta-Analyses extension for Protocols; QALYs: quality-adjusted life years; SF36: 36-Item Short Form Health Survey; SG: standard gamble; SPIRIT: Standard
Protocol Items: Recommendations for Interventional Trials; TTO: time tradeoff; VAS: visual analogue scale.

\section{Competing interests}

The authors declare that they have no competing interests.

\section{Authors' contributions}

The study was conceived by FC-L and MR and developed with critical input from AA-A, AG-A, CC, DG-B, RA-B, EB-D, SP, RT-S and BH. FC-L coordinated the study, conducted the literature search, screened citations and full-text articles, abstracted the data, analysed the data, generated the tables and figures, interpreted the results and wrote the manuscript. MR designed the study, screened citations and full-text articles, abstracted the data, analysed the data, interpreted the results and wrote the manuscript. AA-A and RA-B helped with the literature search, screened the citations, interpreted the results and commented on the manuscript for important intellectual content. AG-A, CC, DG-B, EB-D, SP, RT-S and BH interpreted the results and wrote and edited the final manuscript. FC-L and MR accept full responsibility for the finished article, had access to all of the data, and controlled the decision to publish. All authors read and approved the final manuscript.

\section{Acknowledgements}

This study received no specific funding. FC-L and RT-S are partially funded by Generalitat Valenciana (PROMETEOII/2015/021), INCLIVA and Institute of Health Carlos III/CIBERSAM. MR, EB-D and SP are partially funded by the Spanish Health Services Research on Chronic Patients Network (REDISSEC). $\mathrm{BH}$ is supported by a New Investigator Award from the Canadian Institutes of Health Research and the Drug Safety and Effectiveness Network.

The authors are pleased to acknowledge Dr. Oliver Rivero-Arias (University of Oxford, Oxford, UK), who provided valuable feedback and advice throughout the study. We would like to acknowledge the editor, Dr. lan Shemilt, and the peer reviewers, Dr. Andrew Booth and Dr Raúl Palacio-Rodríguez, for their helpful comments on our submitted manuscript.

\section{Author details \\ ${ }^{1}$ Fundación Instituto de Investigación en Servicios de Salud, Valencia, Spain. ${ }^{2}$ Department of Medicine, University of Valencia/INCLIVA Health Research Institute and CIBERSAM, Valencia, Spain. ${ }^{3}$ Clinical Epidemiology Program, Ottawa Hospital Research Institute (OHRI), Ottawa, ON, Canada. ${ }^{4}$ Instituto Aragonés de Ciencias de la Salud (I + CS), Red de Investigación en Servicios de Salud en Enfermedades Crónicas (REDISSEC), Zaragoza, Spain. ${ }^{5}$ Department of History of Science and Documentation, University of Valencia, Valencia, Spain. ${ }^{6}$ CIBERESP, Institut d'Investigació Biomèdica (IIB) Sant Pau, Barcelona, Spain. ${ }^{7}$ Evidence Synthesis Group, Cornerstone Research Group, Burlington, ON, Canada. ${ }^{8}$ Division of Pharmacoepidemiology and Pharmacovigilance, Spanish Medicines and Healthcare Products Agency (AEMPS), Madrid, Spain. ${ }^{9}$ Ingenio-Spanish National Research Council (CSIC) and UISYS-University of Valencia, Valencia, Spain. ${ }^{10} \mathrm{FISABIO-Salud} \mathrm{Pública,}$ Red de Investigación en Servicios de Salud en Enfermedades Crónicas (REDISSEC), Valencia, Spain. ${ }^{11}$ School of Epidemiology, Public Health and Preventive Medicine, Faculty of Medicine, University of Ottawa, Ottawa, ON, Canada.}

Received: 18 September 2015 Accepted: 22 December 2015

Published online: 07 January 2016

\section{References}

1. Mason J, Drummond M. Reporting guidelines for economic studies. Health Econ. 1995;4:85-94.

2. Drummond MF. A reappraisal of economic evaluation of pharmaceuticals. Science or marketing? Pharmacoeconomics. 1998;14:1-9.

3. Rennie D, Luft HS. Pharmacoeconomic analyses: making them transparent, making them credible. JAMA. 2000;283:2158-60

4. Rennie D, Luft HS. Problems in pharmacoeconomic analyses. JAMA. 2000; 284:1922-4

5. Chan AW, Altman DG. Epidemiology and reporting of randomised trials published in PubMed journals. Lancet. 2005;365:1159-62.

6. Hopewell S, Dutton S, Yu LM, Chan AW, Altman DG. The quality of reports of randomised trials in 2000 and 2006: comparative study of articles indexed in PubMed. BMJ. 2010;340:c723. 
7. Moher D, Tetzlaff J, Tricco AC, Sampson M, Altman DG. Epidemiology and reporting characteristics of systematic reviews. PLoS Med. 2007;4, e78.

8. Hutton B, Salanti G, Chaimani A, Caldwell DM, Schmid C, Thorlund K, et al. The quality of reporting methods and results in network meta-analyses: an overview of reviews and suggestions for improvement. PLoS One. 2014;9: e92508.

9. Elixhauser A, Luce BR, Taylor WR, Reblando J. Health care CBA/CEA: an update on the growth and composition of the literature. Med Care. 1993;31: JS1-JS11. JS18-149.

10. Elixhauser A, Halpern M, Schmier J, Luce BR. Health care CBA and CEA from 1991 to 1996: an updated bibliography. Med Care. 1998;36:MS1-9. MS18-147.

11. Neumann PJ, Stone PW, Chapman RH, Sandberg EA, Bell CM. The quality of reporting in published cost-utility analyses, 1976-1997. Ann Intern Med. 2000;132:964-72.

12. Neumann PJ, Greenberg D, Olchanski NV, Stone PW, Rosen AB. Growth and quality of the cost-utility literature, 1976-2001. Value Health. 2005;8:3-9.

13. Rosen AB, Greenberg D, Stone PW, Olchanski NV, Neumann PJ. Quality of abstracts of papers reporting original cost-effectiveness analyses. Med Decis Making. 2005;25:424-8.

14. Neumann PJ, Fang CH, Cohen JT. 30 years of pharmaceutical cost-utility analyses: growth, diversity and methodological improvement. Pharmacoeconomics. 2009;27:861-72.

15. Jefferson T, Demicheli V, Vale L. Quality of systematic reviews of economic evaluations in health care. JAMA. 2002;287:2809-12.

16. Hutter MF, Rodríguez-Ibeas R, Antonanzas F. Methodological reviews of economic evaluations in health care: what do they target? Eur J Health Econ. 2014;15:829-40.

17. García-Altés A. Twenty years of health care economic analysis in Spain: are we doing well? Health Econ. 2001;10:715-29.

18. Oliva J, Del Llano J, Sacristán JA. Analysis of economic evaluations of health technologies performed in Spain between 1990 and 2000. Gac Sanit. 2002; 16 Suppl 2:2-11.

19. Rodriguez JM, Paz S, Lizan L, Gonzalez P. The use of quality-adjusted lifeyears in the economic evaluation of health technologies in Spain: a review of the 1990-2009 literature. Value Health. 2011;14:458-64.

20. Rodríguez Barrios JM, Pérez Alcántara F, Crespo Palomo C, González García $P$, Antón De Las Heras E, Brosa Riestra M. The use of cost per life year gained as a measurement of cost-effectiveness in Spain: a systematic review of recent publications. Eur J Health Econ. 2012;13:723-40.

21. Rovira J, Antoñanzas F. Economic analysis of health technologies and programmes. A Spanish proposal for methodological standardisation. Pharmacoeconomics. 1995:8:245-52.

22. López-Bastida J, Oliva J, Antoñanzas F, García-Altés A, Gisbert R, Mar J, et al. Spanish recommendations on economic evaluation of health technologies. Eur J Health Econ. 2010;11:513-20.

23. Catalá-López F, Alonso-Arroyo A, Aleixandre-Benavent R, Ridao M, Bolaños M, García-Altés A, et al. Coauthorship and institutional collaborations on costeffectiveness analyses: a systematic network analysis. PLoS One. 2012;7:e38012.

24. Catalá-López F, García-Altés A. Economic evaluation of healthcare interventions during more than 25 years in Spain (1983-2008). Rev Esp Salud Publica. 2010;84:353-69.

25. Moher D, Liberati A, Tetzlaff J, Altman DG. PRISMA group preferred reporting items for systematic reviews and meta-analyses: the PRISMA statement. Ann Intern Med. 2009;151:264-9. W64.

26. Valderas JM, Mendivil J, Parada A, Losada-Yáñez M, Alonso J. Development of a geographic filter for PubMed to identify studies performed in Spain. Rev Esp Cardiol. 2006:59:1244-51.

27. Gold MR, Siegel JE, Russell LB, Weinstein MC. Cost-effectiveness in health and medicine. Oxford: Oxford University Press; 1996.

28. Chan AW, Tetzlaff JM, Altman DG, Laupacis A, Gøtzsche PC, Krleža-Jerić K, et al. SPIRIT 2013 statement: defining standard protocol items for clinical trials. Ann Intern Med. 2013;158:200-7.

29. Moher D, Shamseer L, Clarke M, Ghersi D, Liberati A, Petticrew M, et al. PRISMA-P group. Preferred reporting items for systematic review and meta-analysis protocols (PRISMA-P) 2015 statement. Syst Rev. 2015;4:1.

30. Rothwell PM. External validity of randomised controlled trials: "to whom do the results of this trial apply?". Lancet. 2005;365:82-93.

31. Schulz KF, Altman DG, Moher D, CONSORT Group. CONSORT 2010 statement: updated guidelines for reporting parallel group randomized trials. Ann Intern Med. 2010;152:726-32.
32. Moher D, Hopewell S, Schulz KF, Montori V, Gøtzsche PC, Devereaux PJ, et al. CONSORT 2010 explanation and elaboration: updated guidelines for reporting parallel group randomised trials. BMJ. 2010;340:C869.

33. Briggs $\mathrm{A}$, Sculpher M, Claxton K. Decision modeling for health economics evaluation. Oxford: Oxford University Press; 2006.

34. Dwan K, Altman DG, Clarke M, Gamble C, Higgins JP, Sterne JA, et al. Evidence for the selective reporting of analyses and discrepancies in clinical trials: a systematic review of cohort studies of clinical trials. PLoS Med. 2014; 11:e1001666.

35. Saini P, Loke YK, Gamble C, Altman DG, Williamson PR, Kirkham JJ. Selective reporting bias of harm outcomes within studies: findings from a cohort of systematic reviews. BMJ. 2014;349:96501.

36. Kirkham JJ, Dwan KM, Altman DG, Gamble C, Dodd S, Smyth R, et al. The impact of outcome reporting bias in randomised controlled trials on a cohort of systematic reviews. BMJ. 2010:340:c365.

37. Hopewell S, Loudon K, Clarke MJ, Oxman AD, Dickersin K. Publication bias in clinical trials due to statistical significance or direction of trial results. Cochrane Database Syst Rev. 2009;1:MR000006.

38. Bell CM, Urbach DR, Ray JG, Bayoumi A, Rosen AB, Greenberg D, et al. Bias in published cost effectiveness studies: systematic review. BMJ. 2006;332: 699-703.

39. von Elm E, Altman DG, Egger M, Pocock SJ, Gøtzsche PC, Vandenbroucke $J P$, et al. The Strengthening the Reporting of Observational Studies in Epidemiology (STROBE) statement: guidelines for reporting observational studies. PLoS Med. 2007:4:e296.

40. Husereau D, Drummond M, Petrou S, Carswell C, Moher D, Greenberg D, et al. CHEERS Task Force. Consolidated health economic evaluation reporting standards (CHEERS) statement. BMC Med. 2013;11:80.

41. Guidelines for the economic evaluation of health technologies: Canada [3rd Edition]. Ottawa: Canadian Agency for Drugs and Technologies in Health; 2006. Available at: http://www.cadth.ca/media/pdf/186_ EconomicGuidelines_e.pdf

42. Ramsey S, Willke R, Briggs A, Brown R, Buxton M, Chawla A, et al. Good research practices for cost-effectiveness analysis alongside clinical trials: the ISPOR RCT-CEA Task Force report. Value Health. 2005;8:521-33.

43. Weinstein MC, O'Brien B, Hornberger J, Jackson J, Johannesson M, McCabe $C$, et al. Principles of good practice for decision analytic modeling in healthcare evaluation: report of the ISPOR Task Force on Good Research Practices-Modeling Studies. Value Health. 2003;6:9-17.

44. Drummond MF, Jefferson TO. Guidelines for authors and peer reviewers of economic submissions to the BMJ. The BMJ Economic Evaluation Working Party. BMJ. 1996;313:275-83.

45. Siegel JE, Weinstein MC, Russell LB, Gold MR. Recommendations for reporting cost-effectiveness analyses. Panel on cost-effectiveness in health and medicine. JAMA. 1996;276:1339-41

46. Task Force on Principles for Economic Analysis of Health Care Technology. Economic analysis of health care technology. A report on principles. Ann Intern Med. 1995;123:61-70.

47. Turner L, Shamseer L, Altman DG, Schulz KF, Moher D. Does use of the CONSORT Statement impact the completeness of reporting of randomised controlled trials published in medical journals? A Cochrane review. Syst Rev. 2012;1:60.

48. Panic N, Leoncini E, de Belvis G, Ricciardi W, Boccia S. Evaluation of the endorsement of the preferred reporting items for systematic reviews and meta-analysis (PRISMA) statement on the quality of published systematic review and meta-analyses. PLoS One. 2013;8:e83138.

49. Chalmers I, Glasziou P. Avoidable waste in the production and reporting of research evidence. Lancet. 2009:374:86-9.

50. Ioannidis JP, Greenland S, Hlatky MA, Khoury MJ, Macleod MR, Moher D, et al. Increasing value and reducing waste in research design, conduct, and analysis. Lancet. 2014;383:166-75.

51. Lim ME, Bowen JM, O'Reilly D, McCarron CE, Blackhouse G, Hopkins R, et al. Impact of the 1997 Canadian guidelines on the conduct of Canadian-based economic evaluations in the published literature. Value Health. 2010;13:328-34. 